Topiques, études satoriennes

Topoï Studies, Journal of the SATOR

\title{
La consommation de chocolat et ses topoï dans la fiction des Lumières
}

\section{Élodie Ripoll}

Volume 5, 2021

Le manger et le boire dans la fiction narrative

URI: https://id.erudit.org/iderudit/1081527ar

DOI: https://doi.org/10.7202/1081527ar

See table of contents

Publisher(s)

SATOR, Société d'Analyse de la Topique Romanesque d'Ancien Régime

ISSN

2369-4831 (digital)

Explore this journal

Cite this article

Ripoll, É. (2021). La consommation de chocolat et ses topoï dans la fiction des Lumières. Topiques, études satoriennes / Topoï Studies, Journal of the SATOR, 5, 121-142. https://doi.org/10.7202/1081527ar
Article abstract

This article investigates chocolate in Ancien Régime society through a selection of treatises, dictionaries, and novels from the Enlightenment. These texts provide valuable information on its benefits, preparation, and consumption revealing new dietary as well as social rituals, closely linked to the libertine imagination. In addition, the novels inform the evolution of descriptive practices. The analysis of short excerpts enables us to propose a few topoi, such as "to take one's chocolate," "to invite to take chocolate," "to feel pleasure with chocolate" or "(to attempt) to administer poison or narcotic in chocolate."
This document is protected by copyright law. Use of the services of Érudit (including reproduction) is subject to its terms and conditions, which can be viewed online.

https://apropos.erudit.org/en/users/policy-on-use/ 


\section{La consommation de chocolat et ses topoï dans la fiction des Lumières}

Élodie Ripoll

Universität Stuttgart

Le Mercure Galant de novembre 1711 s'ouvre sur « un article d'une érudition très profonde ${ }^{1} »$ consacré à la «science du chocolat ${ }^{2} »$. La revue file la métaphore : à la liste des ingrédients succède une «méthode exacte pour composer un chocolat très agréable et très $\operatorname{sain}^{3} »$, liant plaisir et santé. Le matériel requis est décrit, tout comme les étapes et les durées de préparation. Après une dizaine de pages techniques, les anecdotes s'enchaînent sur sa consommation en Espagne et dans les pays du Nord, et surtout sur ses nombreux bienfaits, agrémentés de conseils pratiques, comme boire «un verre d'eau afin de n'en être point échauffé ${ }^{4} »$. Cet article témoigne de ce nouveau goût qui marque le XVIII ${ }^{\mathrm{e}}$ siècle. Boisson à la mode, synonyme de luxe et d'oisiveté lorsqu'elle est consommée par des libertins, mais aussi aliment-médicament voire aliment-miracle ${ }^{5}$ aux multiples propriétés et aux multiples controverses, car la « science du chocolat», précise le Mercure Galant, « a cela de commun avec les autres [sciences] qu'elle sera toujours sujette à dispute [car] toute composition où il entre plusieurs drogues, ne saurait contenter tout le monde ${ }^{6} »$.

Les romans témoignent-ils de ce nouveau goût à la mode ? Prennent-ils parti pour ou contre le chocolat ? Sont-ils riches en détails sur sa préparation ou sa dégustation ? Est-il enrichi de cannelle, de vanille, de musc, de poivre ? Ces passages évoluent-ils au cours du siècle à mesure que la description se développe dans le roman?

\footnotetext{
${ }^{1}$ Mercure galant, novembre 1711, p. 4.

${ }^{2}$ Ibid.

${ }^{3}$ Ibid.

${ }^{4}$ Ibid., p. 18.

${ }^{5}$ Cf. Stéphanie Paternotte et Pierre Labrude, «Le chocolat dans quelques ouvrages français de pharmacie et de médecine des XVII ${ }^{\mathrm{e}}$, XVIII ${ }^{\mathrm{e}}$ et XIX ${ }^{\mathrm{e}}$ siècles. Ses effets fastes et néfastes avérés ou supposés », 2003, p. 197 ; Alice Peeters, "Controverses sur les vertus du cacao et les manières de préparer le chocolat $\left(\mathrm{XVI}^{\mathrm{e}}-\mathrm{XVIII} \mathrm{e}^{\mathrm{e}}\right.$ siècle) », 1979, p. 212.

${ }^{6}$ Mercure galant, novembre 1711, p. 4.
} 
Après un état des lieux de la pharmacopée du chocolat sur ses usages et ses contreindications, nous enquêterons sur la production romanesque au siècle des Lumières. Il s'agira ensuite de répertorier les topoï liés à sa consommation autour de trois étapes : la préparation, la dégustation et l'empoisonnement, en analysant leurs configurations narratives et leur évolution au cours du siècle à l'aune des travaux consacrés à l'histoire des aliments, l'histoire des sens et à la culture matérielle.

\section{Le chocolat dans la société d'Ancien Régime}

Le «XVIII ${ }^{\mathrm{e}}$ siècle fut celui de l'apothéose du chocolat, en particulier sous la Régence $^{7} »$. Fernand Braudel constate qu'à Paris en 1768, « les grands en prennent quelquefois, les vieux souvent, le peuple n'en boit jamais ${ }^{8} »$. S'il s'agit d'une boisson d'aristocrates (les bourgeois préférant semble-t-il le café ${ }^{9}$ ), Diderot explique dans l'Encyclopédie que le chocolat est « si bon marché que la tasse ne revient presqu'à un sou $^{10}$ ».

La culture matérielle témoigne en revanche de réels changements dès le XVII ${ }^{\mathrm{e}}$ siècle. «Tout un art se développa autour de la préparation et de la consommation du chocolat: moussoirs, chocolatières, tasses ${ }^{11} »$, la chocolatière dont parle Mme de Sévigné ${ }^{12}$, le service à chocolat qui comporte une petite tasse en porcelaine, la « tasse trembleuse ${ }^{13} »$. De nombreux tableaux représentent ces objets comme Le Déjeuner (1739) de Boucher (Fig. 1), La Belle Chocolatière (1743) de Jean-Étienne Liotard (Fig. 2), La Tasse de chocolat (1768) de JeanBaptiste Charpentier (Fig. 3) ou encore La Crainte (1769) de Jean-Baptiste Le Prince (Fig. 4).

Parallèlement,

on vit apparaître au XVIII ${ }^{\mathrm{e}}$ siècle dans le commerce [...] des mélanges tout préparés de pâte de cacao, de sucre, de cannelle, de vanille, parfois d'autres ingrédients. [...] Ces pains de cacao déjà aromatisés prirent le nom de chocolat, car il n'y avait plus qu'à en râper la quantité nécessaire, y ajouter de l'eau et faire chauffer ${ }^{14}$.

\footnotetext{
${ }^{7}$ Alice Peeters, « Boire le chocolat », 1989, §8.

${ }^{8}$ Fernand Braudel, Civilisation matérielle, économie et capitalisme, XV $V^{e} X V I I I^{e}$ siècle, t. 1, 1979, p. 214 ; voir aussi Patrick Rambourg, « De la consommation des boissons exotiques aux XVII et XVIII ${ }^{\mathrm{e}}$ siècles », 2015, p. 58.

${ }^{9}$ Cf. Sophie D. Coe \& Michael D. Coe, Généalogie du chocolat, 1998, p. 133.

${ }^{10}$ Diderot, art. « CHOCOlat [ECONOMIE DOMESTIQUe / DiÈTE] », 1753, p. 360.

${ }^{11}$ Alice Peeters, « Boire le chocolat », 1989, §8.

${ }^{12}$ Mme de Sévigné, Correspondance, 1972, t. 1, p. 157.

${ }^{13}$ Sophie D. Coe \& Michael D. Coe, Généalogie du chocolat, 1998, p. 92. Voir aussi Guillaume Séret, « Des porcelaines pour sublimer le goût », 2015, p. 85-95.

${ }^{14}$ Alice Peeters, « Controverses sur les vertus du cacao... », 1979, p. 202 sq.
} 
Comme le précise Jean-Claude Bonnet, le « chocolat en tablettes est une nouveauté qui n'est pas tant conçue comme une friandise que comme un condensé pharmaceutique » car « [1]es

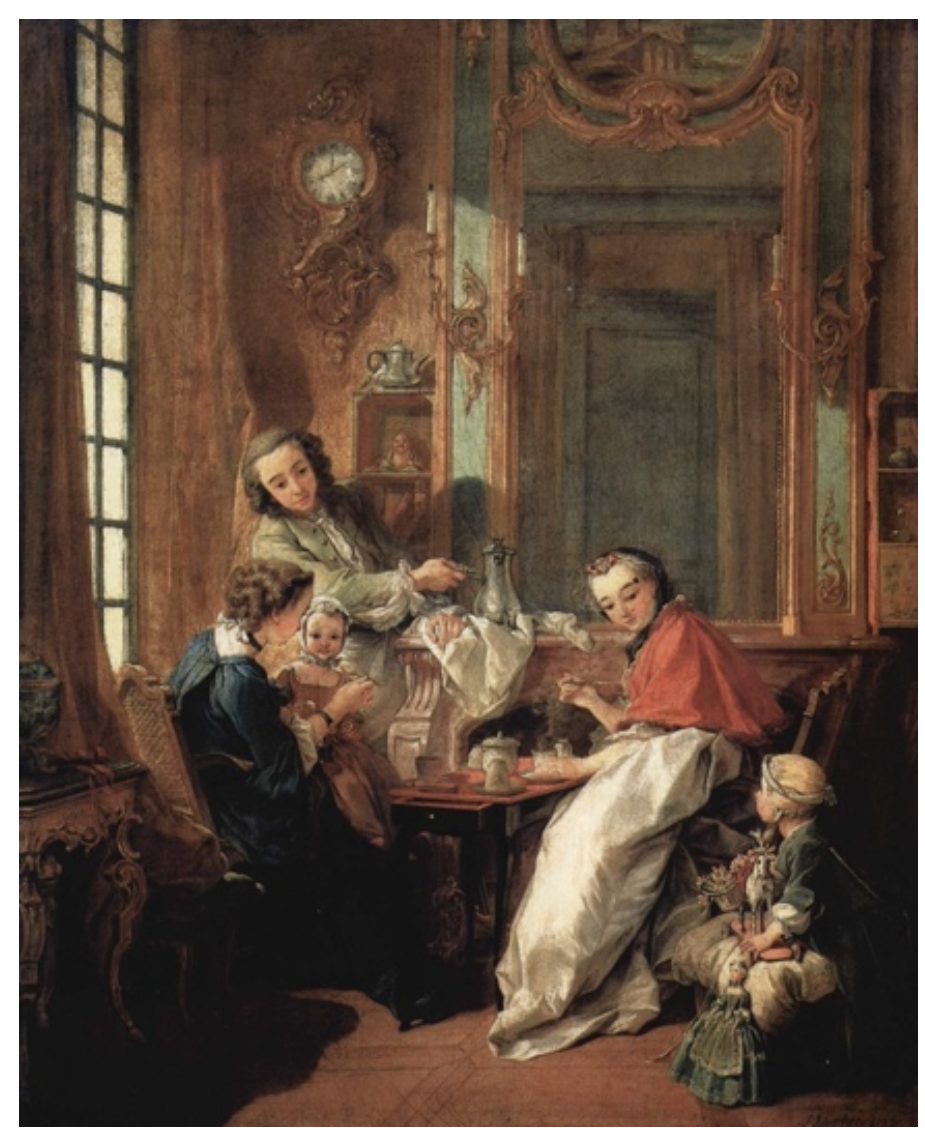

Figure 1: François Boucher, Le Déjeuner, 1739, $81 \mathrm{~cm}$ x $65 \mathrm{~cm}$, huile sur toile, Paris, Musée du Louvre.

pharmaciens s'occupent de pastillage, torréfient le café, [...] font le cacao » et forment «un seul et même corps avec les épiciers ${ }^{15}{ }^{\prime \prime}$.

Les « marchand $[\mathrm{s}]$ droguiste[s] et épicier[s]» (puisque c'est le même corps de métier) ont livré de nombreux témoignages sur leur activité qui soulignent avec justesse les frontières troubles entre pharmacopée et stratégies marchandes. L'un d'eux, Pierre Pomet, publie en 1694 une Histoire générale des drogues illustrée dans laquelle il traite séparément le cacao et le chocolat mais n'évoque aucun de ses bienfaits pharmacologiques ni même sa préparation ; en revanche, d'amples détails sont donnés sur la qualité de son chocolat ${ }^{16}$. Le chocolat est d'abord un objet de commerce et son traité une forme de publicité.

\footnotetext{
${ }^{15}$ Jean-Claude Bonnet, « Le réseau culinaire dans l'Encyclopédie », 1976, p 905 sq.

${ }^{16}$ « Nous vendons de plusieurs sortes de Chocolat, qui ne different que suivant leur beauté \& bonté, \& suivant les drogues dont il est composé \& les pays où il a été fabriqué. Et le meilleur Chocolat $\&$ le plus beau, est celuy que nous faisons [...], surtout quand il a été fait avec le gros Caraque, \& que l'on y a employé de beau sucre, de bonne
} 


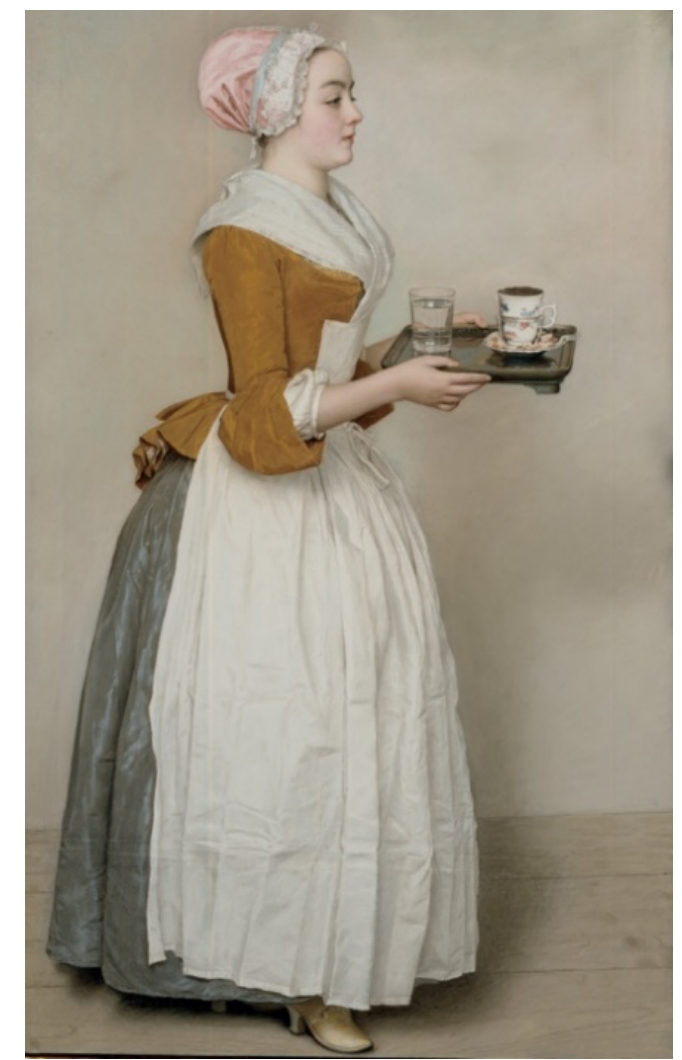

Figure 2: Jean-Étienne Liotard, La Belle Chocolatière, $1743,82,5 \mathrm{~cm} \times 52,5 \mathrm{~cm}$, pastel sur parchemin, Dresde, Gemäldegalerie Alte Meister.

Quatre ans plus tard, en 1698, le Traité universel des drogues simples de Nicolas Lémery s'étend sur sa préparation - à laquelle on peut rajouter « un ou deux jaunes d'œufs frais » et du « lait au lieu d'eau »-ainsi que sur ses « vertus ${ }^{17} »$ :

Le chocolat en quelque manière qu'il soit pris, est un bon restaurant propre pour rappeler les forces abattuës, \& pour exciter de la vigueur ; il résiste à la malignité des humeurs il fortifie l'estomac, le cerveau, \& les autres parties vitales ; il adoucit les serositez trop acres qui descendent du cerveau sur la poitrine ; il excite la digestion, il abat les fumées du vin ${ }^{18}$.

Les vertus du chocolat sont parfois dues aux autres épices entrant dans sa composition. Ainsi le Dictionnaire raisonné universel de matière médicale précise que

[1]es fruits du cacao nourrissent beaucoup, ils rafraîchissent en même temps; ils adoucissent \& calment [...] : mais la vanille \& la cannelle fortifient l'estomac, échauffent,

Canelle, \& de belles \& bonnes Vanilles. [...] c'est une chose impossible de donner de bon Chocolat à bon marché [...] ». Pierre Pomet, Histoire générale des drogues, 1694, p. 206.

${ }^{17}$ Nicolas Lémery, Traité universel des drogues simples, 1723, p. 228 pour les trois citations.

${ }^{18}$ Idem. 
aident à la digestion, $\&$ raniment les esprits. Le chocolat nourrit aussi beaucoup, fortifie l'estomac, ranime les esprits, rétablit les forces abattues ; c'est pourquoi il est d'une grande utilité pour ceux qui se sont épuisés avec les femmes, \& pour ceux qui se livrent à de violents exercices ${ }^{19}$.

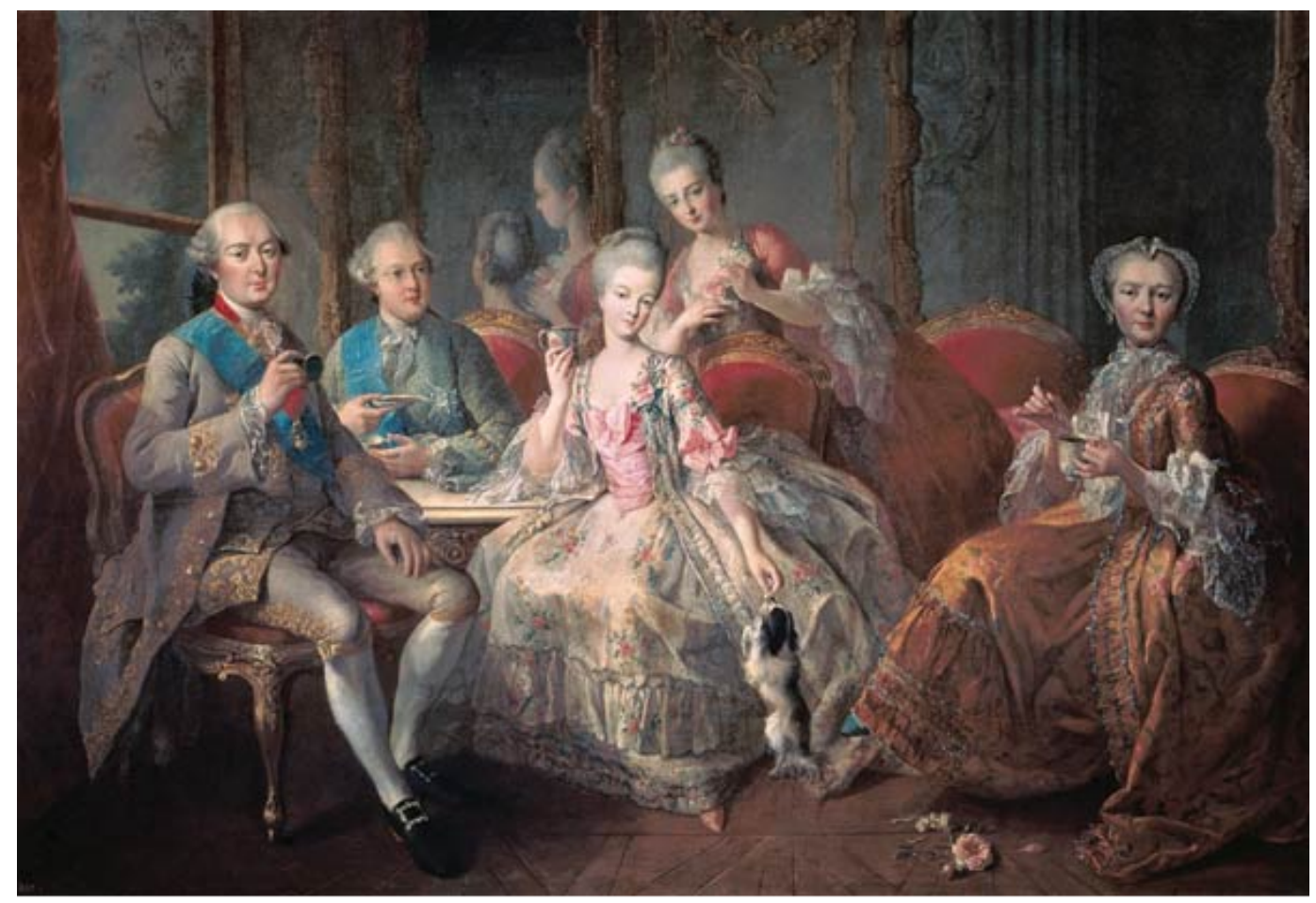

Jean-Baptiste Charpentier, Le Vieux, La Famille du duc de Penthièvre en 1768 dit La Tasse de Chocolat, 1768, $176 \mathrm{~cm} \times 256 \mathrm{~cm}$, huile sur toile, Versailles, châteaux de Versailles et de Trianon.

L'on retrouve des formulations similaires, avec un détail supplémentaire, plutôt amusant : le chocolat est un restauratif qui s'adresse d'abord à un public masculin, mais pas seulement. Les auteurs poursuivent leurs recommandations de la façon suivante :

Le chocolat est bon pour la poitrine ; on en recommande l'usage aux phtisiques, préparé avec le lait; mais il ne faut pas qu'il y ait de vanille dans la composition : il donne un suc nourricier \& doux, qui a la vertu d'émousser l'acrimonie des humeurs. [...] Le chocolat au lait $[\ldots]$ est un excellent déjeuner pour les personnes en consomption.

Il est encore salutaire aux vieillards, aux convalescents, aux personnes délicates \& à ceux qui se relèvent d'une longue maladie. Il ne convient point aux gens de lettres, ni aux hypochondriaques. Les personnes auxquelles il cause des nausées, doivent aussi s'en abstenir. On ne soit cependant pas en faire un trop fréquent usage, parce qu'il épaissit le sang, \& le rend incapable de circuler; d'où il s'ensuit des fievres, l'apoplexie, l'inflammation des visceres ${ }^{20}$.

\footnotetext{
${ }^{19}$ Jean Goulin et Labeyrie, Dictionnaire raisonné universel de matière médicale, t. 2, 1773, p. 67 ; mes italiques.

${ }^{20}$ Ibid., p. 67 sq.
} 
Le chocolat a donc au moins autant de vertus que de contre-indications : fortifiant, favorisant la digestion, aphrodisiaque... Cela explique peut-être pourquoi Gabriel-François Venel, dans l'autre article « chocolat» de l'Encyclopédie, y voit surtout une boisson qui « ne mérite ni tout le bien ni tout le mal qu'on en a $\operatorname{dit}^{21} \gg$.

D'autres documents renseignent également sur certaines croyances comme les relations de voyage ou encore les traités sur l'alimentation, comme le Traité des aliments de Louis Lémery qui s'appuie vraisemblablement sur les travaux de son père :

Le Chocolat nourrit beaucoup ; il est fortifiant, restaurant, propre pour réparer les forces abatues, \& pour donner de la vigueur. Il aide à la digestion ; il adoucit les humeurs âcres qui tombent sur la poitrine; il abat les fumées du Vin; il excite les ardeurs de Vénus, \& il résiste à la malignité des humeurs ${ }^{22}$.

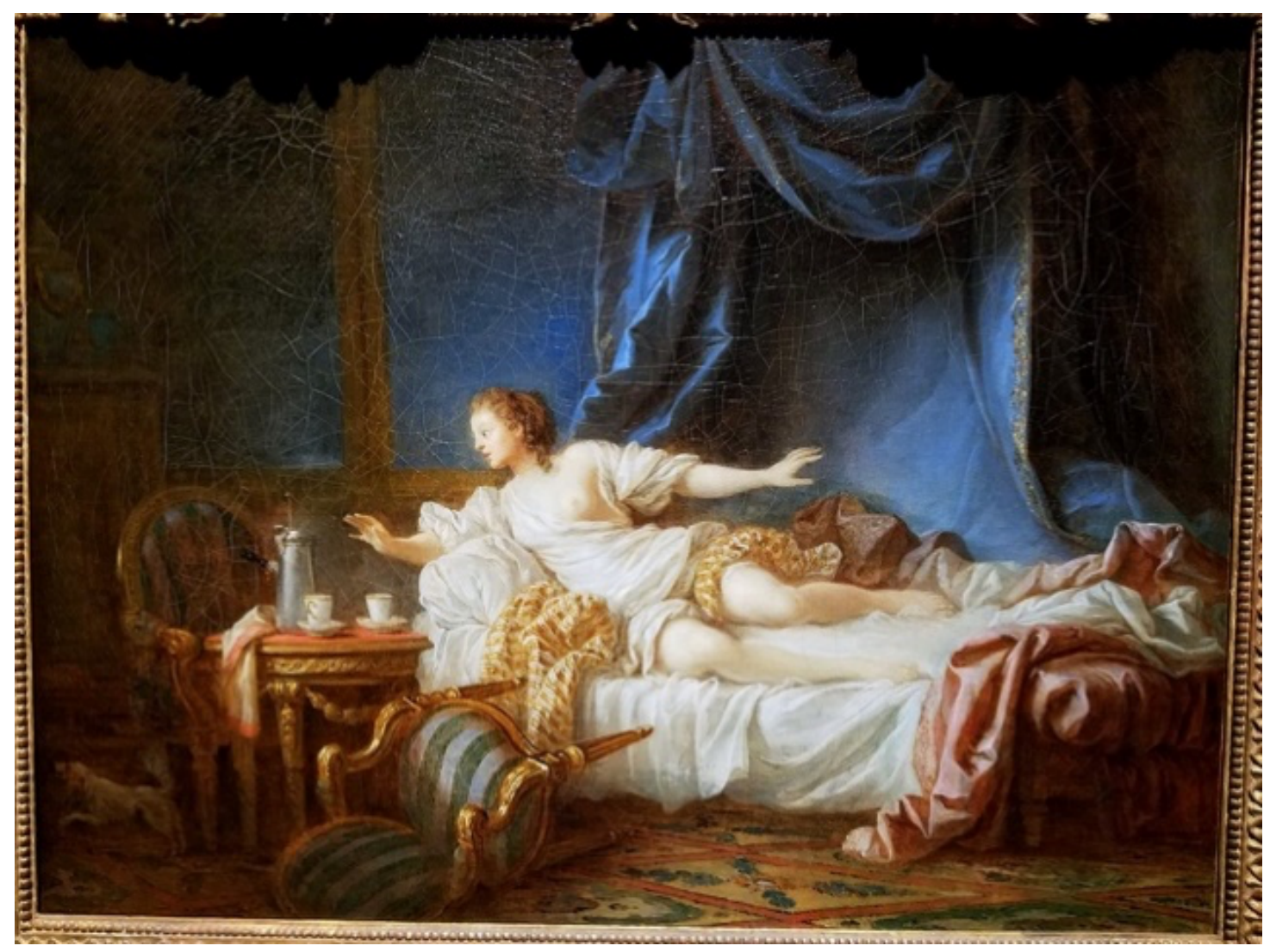

Figure 4: Jean-Baptiste Le Prince, La Crainte, 1769, $50 \mathrm{~cm} \times 64 \mathrm{~cm}$, huile sur toile, Toledo, Museum of Art.

${ }^{21}$ Gabriel-François Venel, art. CHOCOLAT, Encyclopédie, 1753, vol. III, p. 360.

${ }^{22}$ Louis Lémery, Traité des aliments, [1702] 1705, p. 531 sq. Les passages en italique indiquent les reprises du Traité universel des drogues simples de Nicolas Lémery. 
Dans tous ces traités, les propriétés pharmacologiques du chocolat sont secondaires face aux longues digressions consacrées à sa préparation : le chocolat est d'abord un objet de gourmandise qui commence dès la lecture de la recette. « Nous sommes loin, constate JeanClaude Bonnet, des recettes d'aujourd'hui faites de concision, de proportions impératives, de sécheresse technique ${ }^{23} »$. L'intérêt pour sa préparation se limite cependant aux recettes, parfois aux planches techniques de l'Encyclopédie consacrées aux confiseurs ${ }^{24}$, rares sont les voix qui, comme Bernardin de Saint-Pierre, dénonce l'esclavage lié à sa production ${ }^{25}$.

\section{Modalités de la consommation de chocolat dans le roman des Lumières}

Les personnages de romans prennent leur chocolat chez eux (dans leur chambre ${ }^{26}$, voire à leur toilette ${ }^{27}$ ou sur leur sopha ${ }^{28}$ ), à l'extérieur (chez des proches, des connaissances), voire dans des cafés ${ }^{29}$, le lieu en vogue au XVIII ${ }^{\mathrm{e}}$ siècle $^{30}$. Le chocolat peut accompagner un repas, comme ici dans Gil Blas :

Le cuisinier, l'aide de cuisine et le marmiton n'étaient pas oisifs pendant ce temps-là ; ils travaillaient à nous préparer un repas supérieur à celui que nous avions fait; et nous fûmes dans le dernier étonnement, lorsqu'étant entrés dans la même salle où nous avions dîné, nous vîmes mettre sur la table un plat de quatre perdreaux rôtis, avec un civet de lapin d'un côté, et un chapon en ragoût de l'autre. Ils nous servirent ensuite pour entremets des oreilles de cochon, des poulets marinés et du chocolat à la crème ${ }^{31}$.

Le chocolat peut tenir « ordinairement lieu de dîner ${ }^{32}$ », mais le plus souvent, il se déguste pendant une collation. Jacques de Varenne, dans les Mémoires du chevalier de Ravanne, évoque « l'heure du chocolat ${ }^{33}$ » sans plus de précision. On apprend chez Beaumarchais qu'être invité « au chocolat », c'est être invité à neuf heures ${ }^{34}$, mais chez Mirabeau c'est à dix heures ${ }^{35}$, voire

\footnotetext{
${ }^{23}$ Jean-Claude Bonnet, « Le réseau culinaire dans l'Encyclopédie », 1976, p. 908.

${ }^{24}$ Voir « Confiseur. Chocolat et Moules pour les Fromages », ConfiseUR. PL. V, Encyclopédie, 1763, Planches

t. III, $s . p$

${ }^{25}$ Bernardin de Saint-Pierre, « Lettre XII », Voyage à l'île de France, 1840, p. 55-58.

${ }^{26}$ Dulaurens, Le Compère Mathieu ou les Bigarrures de l'esprit humain, [1766] 2012, p. 398.

${ }^{27}$ Voltaire, La Princesse de Babylone, [1768] 1954, p. 422.

${ }^{28}$ Lesage, Histoire de Gil Blas de Santillane, [1732] 1987, p. 917.

${ }^{29}$ Jacques de Varenne, Mémoires du chevalier de Ravanne, 1782, t. III, p. 71, 80, 216.

${ }^{30}$ Stéphane Pujol, art. « Cafés », Dictionnaire européen des Lumières, 1997, p. 208 sqq.

${ }^{31}$ Lesage, Histoire de Gil Blas de Santillane, [1732] 1987, p. 1045.

${ }^{32}$ Jacques de Varenne, Mémoires du chevalier de Ravanne, 1782, t. III, p. 59.

${ }^{33}$ Ibid., t. I, p. 271.

${ }^{34}$ Beaumarchais, Mémoires contre M. Goëzmann, [1774] 1828, p. 348.

${ }^{35}$ Mirabeau, Le Libertin de qualité, ou Ma conversion, [1783] 1911, p. 46.
} 
« entre dix et onze $\mathrm{e}^{36} »$ chez Fougeret de Monbron ou encore à « onze heures ${ }^{37} »$ chez Sade. Ces habitudes sont donc assez flottantes. Plus qu'une heure précise, le chocolat est surtout un moment particulier qui renvoie à de nouveaux rituels, tant alimentaires que sociaux. On y est invité, on s'y retrouve, on discute de sujets importants, on s'y fait des confidences.

Le chocolat est en outre un marqueur social, soit de richesse, soit de dignité, ou du moins est-il perçu comme tel. Ainsi, ce personnage de Lesage qui, dit-il, se « fait donner mon chocolat afin que l'on crût par là que je n'étais pas un homme du commun ${ }^{38}$ ». Maîtres et maîtresses de maison l'offrent à leurs invités, il peut même faire partie du salaire. Dans Le Petitfils d'Hercule, une «petite vieille sèche et ridée, bossue par derrière » propose au narrateur « cinq louis par coup, souper, liqueur, chocolat à discrétion ${ }^{39}$ » en échange de ses services qui nécessiteront un restauratif.

Conformément aux discours médicaux existants, le chocolat sert de prélude à toutes sortes d'activités. C'est un fortifiant qui peut remplacer le bouillon ${ }^{40}$, régler « une grande faiblesse d'estomac ${ }^{41} \gg$ et favoriser les efforts de concentration : ainsi cet autre personnage de Lesage boit-il une tasse de chocolat avant de passer « toute la journée à jouer aux échecs ${ }^{42}$ »; de façon plus amusante, l'éditrice des Mémoires de Suzon confie à la fin de la Préface : «Je commençai donc par prendre une tasse de chocolat, pour me donner la force de soutenir une si longue tâche, et je me mis ensuite à lire les Mémoires suivants ${ }^{43}{ }^{4}$. Remarque d'autant plus amusante qu'il s'agit d'un roman libertin - boire du chocolat est donc une précaution avant d'ouvrir un de ces livres qu'on ne lit que d'une main. Dans l'imaginaire libertin, le chocolat, tant prélude que restauratif, encadre pour ainsi dire toute activité : qu'il s'agisse d'une lecture indirecte ou de pratique directe. Dans l'Anti-Justine, on s'en sert même "pour se rincer la bouche ${ }^{44} »$. Ce faisant, le chocolat n'est en aucun cas uniquement requis pour « ceux qui se sont épuisés avec les femmes », ces dernières le consomment autant que les hommes ${ }^{45}$.

Par extension, la consommation de chocolat annonce sinon la débauche, du moins une ambiguïté. C'est le cas dans La Religieuse où Suzanne raconte l'attitude de la mère supérieure :

\footnotetext{
${ }^{36}$ Fougeret de Monbron, Margot la ravaudeuse, [1750] 2000, p. 837.

${ }^{37}$ Sade, Histoire de Juliette, [1797] 1998, p. 541.

${ }^{38}$ Lesage, Histoire de Guzman d'Alfarache, [1732] 1825, p. 73.

${ }^{39}$ Anonyme, Le Petit-fils d'Hercule, [1788] 2005, p. 1086.

${ }^{40}$ Jacques de Varenne, Mémoires du chevalier de Ravanne, 1782, t. II, p. 32.

${ }^{41}$ Ibid., t. III, p. 216.

${ }^{42}$ Lesage, Le Diable boiteux, 1987, p. 465.

${ }^{43}$ Anonyme, Les Mémoires de Suzon, 2005, p. 886.

${ }^{44}$ Restif de la Bretonne, L'Anti-Justine, 2014, p. 270.

${ }^{45}$ Ibid., par exemple.
} 
« on ne lui faisait aucun présent que je ne le partageasse : chocolat, sucre, café, liqueurs, tabac, linge, mouchoirs ${ }^{46} »-$ autant de produits coûteux (et aussi superflus) qui correspondent mal au vœu de pauvreté attendu des religieuses. Le personnage féminin qui ignore (ou feint d'ignorer) les véritables intentions de sa supérieure ne connaît pas non plus les valeurs symboliques de ces présents. L'on peut sans doute ici reprendre une lecture de Michel Bernard à propos de Madame Bovary: «le danger des sucreries» est autant «luxe» et «douceur de la richesse » que corruption lente des mœurs ${ }^{47}$.

D'une manière générale, peu d'attention est accordée au chocolat lui-même. Tout juste apprend-on que certains personnages le servent « copieu[x] » avec deux biscuits ${ }^{48}$, que d'autres ne l'aiment pas « léger ${ }^{49} »$. Est-il alors enrichi de cannelle, de vanille, de musc, de poivre, voire «d'essence d'ambre ${ }^{50} »$ ou d'un « œuf frais entier ${ }^{51} »$ ? Les remarques concernant la préparation elle-même ne dépassent pas l'anecdote comme dans cet extrait de Candide : « [...] deux filles jolies et proprement mises servirent du chocolat qu'elles firent très bien mousser. Candide ne put s'empêcher de les louer sur leur beauté, sur leur bonne grâce et sur leur adresse $^{52}$. » La mention de la mousse n'est que le prétexte à l'éloge de leur savoir-faire : le lecteur en ignore les causes (est-ce de l'œuf frais comme on l'apprend dans l'Encyclopédie ${ }^{53}$ ou de l'huile de coude ?). De même, la qualité du chocolat est d'abord un signe de richesse, on la dit plus qu'on ne la décrit, comme dans Thémidore : «Je lui promis de l'attendre avec une tasse de chocolat qu'il accepta, après que je lui eus persuadé que le mien était préférable à celui dont il usait ordinairement ${ }^{54} »$.

Sa température est parfois mentionnée : si le chocolat est chaud, il est prêt, on peut le déguster $^{55}$. S'il est froid, c'est un drame, comme dans cet extrait de Six semaines de la vie de Faublas : « Tandis qu'il nous montrait dans l'avenir des malheurs imaginaires, un malheur réel venait de nous arriver ; le chocolat s'était refroidi. Jugez de mon chagrin à moi ${ }^{56} »$. Ce chagrin ira crescendo, le chocolat sera renversé :

\footnotetext{
${ }^{46}$ Diderot, La Religieuse, 2004, p. 338.

${ }^{47}$ Voir Michel Bernard, « Madame Bovary ou le danger des sucreries », 1999, p. 41-51.

${ }^{48}$ Jacques de Varenne, Mémoires du chevalier de Ravanne, 1782, t. III, p. 195.

${ }^{49}$ Fougeret de Monbron, Margot la ravaudeuse, 2000, p. 838.

${ }^{50}$ Diderot, art. CHOCOLAT. op. cit.

51 Ibid.

${ }^{52}$ Voltaire, Candide, [1759] 1995, p. 143.

${ }^{53}$ Voir supra note 50.

${ }^{54}$ Godard d'Aucour, Thémidore, [1744] 2000, p. 547.

${ }^{55}$ Louvet de Couvray, Six semaines de la vie de Faublas, [1787] 1965, p. 822.

${ }^{56}$ Ibid., p. 821.
} 
[...] ne croyez pas que je regrette ni le petit meuble démantibulé, ni les porcelaines brisées, ni la chocolatière bossuée, ni mon plus beau jupon gâté ; non, je ne vois que le chocolat coulant à grands flots sur le parquet ${ }^{57}$.

Ce premier panorama ne semble pas propice à une lecture satorienne de la consommation de chocolat: tous les lieux, toutes les heures semblent convenir, permettant à un imaginaire du luxe et de la volupté de se mettre en place.

\section{Une épistémologie des sens}

Cette économie relative de détails ne surprend guère et pourrait être due à cette « hiérarchie des formes » représentative du début du XVIII ${ }^{\mathrm{e}}$ siècle « qui réservait l'évocation de la vie quotidienne et des réalités physiques au style bas ${ }^{58} »$. Le roman des Lumières se méfie encore assez des descriptions et il faudra attendre un « renouveau des pratiques descriptives ${ }^{59}$ » plus large pour assister à une « réhabilitation romanesque du détail ${ }^{60} »$ dès les années 1760 . Ces nouvelles manières d'observer et de décrire sont liées à l'émergence progressive d'une nouvelle épistémologie visuelle, héritière de l'empirisme lockéen, de la revalorisation de l'observation et $\mathrm{du}$ goût pour les sciences naturelles ${ }^{61}$. Ces quelques remarques consacrées au chocolat annoncent en revanche les changements à venir. Elles sont de deux natures : des trivialités dans certains romans (l'exemple du repas copieux dans Gil Blas), un exemple de l'intérêt porté aux perceptions sensorielles dans le roman libertin ${ }^{62}$.

La hiérarchie traditionnelle des sens a longtemps privilégié la vue au détriment de l'odorat, de l'ouïe, du toucher et du goût ${ }^{63}$, les sens de la distance étant généralement valorisés face aux sens du contact - bien que la couleur ait toujours été ambivalente ${ }^{64}$. La philosophie des Lumières réévalue les sens et leurs perceptions dans le sillage de l'empirisme et du

\footnotetext{
${ }^{57}$ Ibid., p. 823.

${ }^{58}$ Michel Delon, « Le détail et l'histoire », 2007, p. 159 sq.

${ }^{59}$ Christof Schöch, La Description double dans le roman des Lumières, 2011, p. 11.

${ }^{60}$ Michel Delon, op. cit., p. 159 sq.

${ }^{61}$ Voir Joanna Stalnaker, The Unfinished Enlightenment. Description in the Age of the Encyclopedia, 2010 ; Evelyn Dueck et Nathalie Vuillemin (éds.), « Der Augen Blödigkeit ». Sinnestäuschungen, Trugwahrnehmungen und visuelle Epistemologie im 18. Jahrhundert, 2016 ; id., Entre l'œil et le monde : dispositifs d'une nouvelle épistémologie visuelle dans les sciences de la nature (1740-1840), 2017.

${ }^{62}$ Je reprends ici le terme utilisé dans l'édition des Romanciers libertins du XVIII ${ }^{e}$ siècle en deux volumes, établie sous la direction de Patrick Wald Lasowski (2005). Pour une définition du roman libertin, on consultera : Jacques Rustin, « Définition et explicitation du roman libertin des Lumières », 1978, p. 26-34 ; Michèle Bokobza Kahan, Libertinage et Folie dans le roman du $18^{e}$ siècle, 2000, p. 5-11 ; Marc André Bernier, Libertinage et figures du savoir, 2001, p. 41-50.

${ }^{63}$ Voir D. M. Levin, Modernity and the Hegemony of Vision, 1993 ; Mark M. Smith, Sensory History, 2007 ;

Géraldine Puccini-Delbey, Le Débat des cinq sens de l'Antiquité à nos jours, 2014.

${ }^{64}$ Voir Élodie Ripoll, Penser la couleur en littérature, 2018.
} 
sensualisme. Diderot écrit à ce sujet dans la Lettre sur les sourds et muets (1751) que « de tous les sens l'œil était le plus superficiel, l'oreille le plus orgueilleux, l'odorat le plus voluptueux, le goût le plus superstitieux et le plus inconstant, le toucher le plus profond et le plus philosophe ${ }^{65} »$. Il dresse ainsi une typologie des sens où l'odorat se voit revalorisé. Le roman libertin fait fi des préjugés traditionnels et joue avec les perceptions sensorielles, quelles qu'elles soient, toutes plus voluptueuses les unes que les autres. Ce faisant, il pratique plus la description que les autres romans de son époque et fait office de précurseur en la matière. Le chocolat est un exemple de choix car il régale plusieurs sens : il est d'abord une odeur que l'on perçoit de loin, une couleur qui parle à la vue, un goût, une texture que l'on sent en buvant. Un plaisir complexe que les personnages se plaisent à évoquer.

Margot, l'héroïne éponyme de Fougeret de Monbron, explique « régale[r] [son] odorat et [son] palais du parfum agréable de ce liquide mousseux ${ }^{66} \gg$. Deux sens secondaires sont ici stimulés, l'odorat et le goût. Les nombreuses recettes détaillées par le Mercure galant ou par l'Encyclopédie nous renseignent sur ces préférences: le «parfum agréable ${ }^{67}$ » peut être composé de vanille, de musc, de poivre, de cannelle, ces épices livrent de précieuses indications ; sur le plan symbolique, elles participent d'un imaginaire du luxe et provoquent d'autres effets physiologiques. La vanille et la cannelle échauffent ${ }^{68}$, le musc suscite l'amour ou l'aversion ${ }^{69}$. Paradoxalement, le goût du chocolat semble aller à rebours des odeurs florales (eau de rose, violette, thym, romarin, lavande) valorisées à l'époque des Lumières au détriment des « odeurs fortes », des « parfums animaux » comme le musc ${ }^{70}$.

Au cours d'une visite à Mme de Dorigny, le héros de Thémidore s'attarde sur ses perceptions olfactives comme celles visuelles, voire tactiles et haptiques des tissus :

Elle me reçut à sa toilette [...]. Les odeurs qui remplissaient les boîtes n'étaient pas fortes et en grande quantité, mais elles étaient douces et répandaient un parfum suave qui embaumait légèrement la chambre et vous flattait délicieusement l'odorat; son linge de nuit garni d'une dentelle petite, mais fine, était travaillé avec goût, sa robe de perse, son jupon de satin piqué ${ }^{71}[\ldots]$.

\footnotetext{
${ }^{65}$ Diderot, Lettre sur les sourds et muets, [1751] 1978, p. 140.

${ }^{66}$ Fougeret de Monbron, Margot la Ravaudeuse, [1750] 2000, p. 838.

${ }^{67}$ Ibid.

${ }^{68}$ Cf. Jaucourt, art. VANILLE 1765, p. 830 et art. CANELLE, 1752, vol. II, p. 592.

${ }^{69}$ Ibid., art. MUSC, 1765, vol. X, p. 878.

${ }^{70} \mathrm{Cf}$. Alain Corbin, Le miasme et la jonquille, 2016, p. 111-118.

${ }^{71}$ Godard d'Aucour, Thémidore, 2000, p. 574.
} 
Restés seuls, ils peuvent s'embrasser «avec transport », « pendant qu'on [leur] préparait un chocolat voluptueux ${ }^{72}$ » dont l'odeur sera plus intense que les parfums initiaux - au-delà de cette gradation, ce chocolat réalisera une synthèse des sens : mêlant le goût, le toucher, l'odorat, il annonce des expériences synesthésiques avant la lettre.

\title{
Le chocolat empoisonné
}

Le chocolat présente un autre intérêt, où se rejoignent imaginaire littéraire et réalité historique : « l'Espagne baroque, et de fait toute l'Europe, fit une [...] découverte fort utile et intéressante: le goût prononcé du chocolat permettait d'y dissimuler efficacement des poisons ${ }^{73} »$. La première association entre chocolat et poison en français figure sans doute dans la Relation du voyage d'Espagne de Mme d'Aulnoy, plus précisément dans la lettre XII où elle évoque le tempérament des Espagnoles et leur désir de vengeance :

\begin{abstract}
Elles mettent tout en usage pour se venger de leurs amans, s'ils les quittent sans sujet : de sorte que les grands attachements finissent d'ordinaire par quelque catastrophe funeste. Par exemple, il y a peu, qu'une femme de qualité ayant lieu de se plaindre de son amant, elle trouva le moment de le faire venir dans une maison, dont elle étoit la maîtresse ; \& aprés lui avoir fait de grands reproches, dont il se deffendit foiblement, parce qu'il les méritoit, elle lui presenta un poignard \& une tasse de chocolat empoisonné, lui laissant seulement la liberté de choisir le genre de mort. Il n'employa pas un moment pour la toucher de pitié : il vit bien qu'elle étoit la plus forte en ce lieu ; de sorte qu'il prit froidement le chocolat, \& n'en laissa pas une goutte. Aprés l'avoir bû [sic] ; il lui dit : Il auroit été meilleur, si vous y aviez mis plus de sucre ; car le poison le rend fort amer : souvenez vous-en pour le premier que vous accommoderez. Les convulsions le prirent presque aussi-tôt ; c'étoit un poison trés-violent, \& il ne demeura pas une heure à mourir. Cette dame, qui l'aimoit encore passionnément, eut la barbarie de ne le pas quitter qu'il ne fut mort $^{74}$.
\end{abstract}

Cette scène présentée comme une anecdote récente reprend certains éléments de l'histoire d'Aliénor d'Aquitaine, épouse d'Henri II, et de sa maîtresse Fair Rosamund : l'épouse légitime pousse la maîtresse au suicide et lui donne le choix entre le poignard et le poison ${ }^{75}$. La scène médiévale est ici actualisée, la composante genrée modifiée - le chocolat est la boisson choisie par la meurtrière, l'amant raille le goût amer du poison que le chocolat ne parvient pas à masquer, l'on apprend également des détails sur ses convulsions et la durée de son agonie.

\footnotetext{
72 Ibid.

${ }^{73}$ Sophie D. Coe \& Michael D. Coe, Généalogie du chocolat, 1998, p. 93.

${ }^{74}$ Madame d'Aulnoy, Relation du voyage d'Espagne, 1691, t. III, p. 110 sq.

${ }^{75}$ Voir Michael R. Evans, Inventing Eleanor. The Medieval and Post-Medieval Image of Eleanor of Aquitaine, 2014 et The Ballad of Fair Rosamund de Thomas Deloney (1612).
} 
Le chocolat empoisonné devient rapidement un topos qui, par un déplacement géographique, « fait italien ${ }^{76} »$ pour reprendre Henri Lafon qui ne développe pourtant aucun exemple. «Tout le monde sait que les Italiens font un grand usage de poison ${ }^{77}$ », précise Sade dans Juliette, poussant Isabel Herrero et Lydia Vasquez à évoquer la «manie du poison ${ }^{78}$ » parmi les stéréotypes des personnages italiens de romans (ou plus exactement des femmes italiennes). Ainsi, dans Candide, la vieille, italienne de naissance, raconte la mort de son amant :

[...] une vieille marquise qui avait été maîtresse de mon prince l'invita à prendre du chocolat chez elle. Il mourut en moins de deux heures avec des convulsions épouvantables ${ }^{79}$.

Plusieurs éléments de cette scène rappellent le récit de Mme d'Aulnoy : l'invitation, l'ancienne maîtresse, le désir de vengeance, les convulsions ainsi que la durée de l'agonie (plus longue), bien que l'empoisonnement soit ici dissimulé. Le ton sommaire adopté dans Candide ne permet pas de longs développements sur le goût du chocolat. À partir de cet exemple, on pourrait déjà proposer le topos « faire boire un chocolat empoisonné » et les toposèmes suivants : l'invitation, la vengeance, la dissimulation, les convulsions, la durée de l'agonie, l'élément italien de couleur locale. Rien toutefois sur le goût du chocolat.

Lorsqu'un témoin direct prend en charge le récit, les détails sur la préparation et la mise en scène de la convivialité sont bien plus riches : tout est fait pour ne pas éveiller les soupçons bien que la profusion de détails contribue paradoxalement à rendre ces scènes inhabituelles pour le lecteur. C'est le cas dans Félicia ou Mes fredaines de Nerciat à propos d'un épisode concernant le chevalier d'Aiglemont, le favori de la narratrice, qui fréquente un jeune Italien, Géronimo Fiorelli, ses deux sœurs, Argentine et Camille, et préfère la première :

Le hasard voulut que d'Aiglemont se trouvant le lendemain de bonne heure chez les Fiorelli, Argentine l'invitât à prendre du chocolat en famille. [...]

Ce fut la rancuneuse Camille, dont on était bien éloigné d'interpréter la perfide joie, qui se chargea de donner les ordres nécessaires. Elle alla trouver l'exécrable duègne, qui se mit aussitôt à l'ouvrage. On convint d'apporter le chocolat tout versé dans quatre tasses, deux blanches, empoisonnées, dont Camille aurait soin de présenter, l'une au chevalier et l'autre à sa sœur ; et deux coloriées, naturelles, dont une serait pour le frère et l'autre pour Camille elle-même. [...] Le crime ainsi concerté, Camille rejoint la compagnie... ${ }^{80}$.

\footnotetext{
${ }^{76}$ Henri Lafon, Les Décors et les choses dans le roman français du dix-huitième siècle de Prévost à Sade, 1992, p. 370 .

${ }^{77}$ Sade, Histoire de Juliette, [1797] 1998, p. 745.

${ }^{78}$ Isabel Herrero \& Lydia Vazquez, « Types nationaux européens dans des œuvres de fiction françaises (17501789)», 1993, p. 122.

${ }^{79}$ Voltaire, Candide, [1759] 1995, p. 77.

${ }^{80}$ Nerciat, Félicia ou Mes fredaines, [1775] 2005, p. 723.
} 
Nous retrouvons ici les toposèmes observés dans Candide : l'occasion conviviale « en famille » destinée à tromper, la vengeance, la mise en scène et le personnage de la « duègne » - variante de la vieille marquise -, adepte des «plus crapuleuses débauches ${ }^{81} »$, « toujours pourvue de poisons subtils ${ }^{82}$ » et qui accessoirement ne « savait pas un mot de français ${ }^{83} »$ - élément de décisif de couleur locale :

La duègne parut avec un front assuré, portant les quatre tasses, sur un plateau. Elle vanta beaucoup la qualité du chocolat, et le talent qu'elle avait de le préparer supérieurement. Puis ayant fait un second voyage, pour apporter des échaudés, elle vit avec joie que chacun avait devant soi, la tasse qui lui était destinée ; on paraissait attendre pour déjeuner que la boisson, qu'on transvasait des tasses dans les soucoupes fût un peu refroidie ${ }^{84}$.

La composition du chocolat reste secrète, les gâteaux ont été choisis à dessein, comme les tasses : le blanc associé spontanément à la pureté est ici la marque du poison. Mais Camille, prise de remords, a alerté ses proches qui décident alors de tendre un piège à la duègne et de remettre en scène la collation :

Cependant Géronimo dit qu'il ne se sentait point d'appétit et remit une des tasses coloriées sur le plateau. L'infâme empoisonneuse, trompée par la couleur, demanda cette tasse, et de la sorte, donna d'elle-même dans un piège qui venait de lui être tendu. Pendant qu'elle avait été dehors, on s'était hâté de substituer proprement au chocolat naturel, qui était en premier lieu dans la tasse coloriée, celui que devait avaler l'un des deux proscrits ${ }^{85}$.

La duègne «aval[e] » ensuite «avec sensualité ${ }^{86}$ » son chocolat. Le poison agit immédiatement : «L'effet fut prompt. D’affreuses convulsions l'annonçaient presque sur-lechamp $^{87}$ ». Comme chez Mme d'Aulnoy et dans Candide, l'on retrouve le même terme de « convulsion », partie intégrante du topos.

Observons à présent quelques scènes d'empoisonnement au chocolat chez Sade. Notre premier exemple est en réalité triple: il s'agit des différentes versions de la tentative d'empoisonnement planifiée par Bressac sur sa mère avec la complicité forcée de Justine dans Les Infortunes de la vertu, Justine ou les Malheurs de la vertu et La Nouvelle Justine. Les trois versions présentent une séquence d'événements similaires. Les motivations bassement vénales

\footnotetext{
${ }^{81}$ Ibid., p. 722 pour les trois citations.

${ }^{82}$ Ibid., p. 723.

${ }^{83}$ Ibid., p. 724.

${ }^{84}$ Ibid., p. 723 sq.

${ }^{85}$ Idem.

${ }^{86}$ Ibid., p. 724.

${ }^{87}$ Idem.
} 
du jeune homme s'accompagnent d'un discours pour les « yeux peu philosophiques » de Justine sur «la destruction de [ses] semblables », légitimant le meurtre, et surtout le meurtre de la mère ${ }^{88}$. Justine feint d'y adhérer, dévoile le complot à Mme de Bressac, empêche la première tentative mais subit le courroux du fils qui se venge et la chasse avant d'assassiner sa mère. Dans les deux premières versions, le poison doit être administré dans du chocolat :

\begin{abstract}
Nous convînmes que dans deux ou trois jours plus ou moins, suivant la facilité que j'y trouverais, je jetterais adroitement un petit paquet de poison dans une tasse de chocolat que la comtesse avait coutume de prendre tous les matins [...]. [...] je fis voir à la comtesse le paquet de poison dont j'étais chargée ; nous en fîmes avaler une légère dose à un chien que nous enfermâmes avec soin et qui mourut au bout de deux heures dans des convulsions épouvantables ${ }^{89}$.
\end{abstract}

Certes, le chien n'avale pas le poison avec du chocolat, mais la durée de deux heures et la formulation des « convulsions épouvantables » montre un lien intertextuel supplémentaire avec Candide qui est étayé par de nombreux passages des Infortunes de la vertu ${ }^{90}$ - lien qui perdure également pour cet extrait dans Justine ou les Malheurs de la vertu ${ }^{91}$. Au-delà de cette réécriture, certains toposèmes sont également présents : la vengeance, la dissimulation, les convulsions, la durée de l'agonie. Dans La Nouvelle Justine en revanche, Sade délaisse le chocolat pour de « l'eau de tilleul ${ }^{92} »$, la boisson que prend désormais Mme de Bressac tous les matins, tandis que le chien agonise «au bout de deux heures, dans d'effroyables convulsions ${ }^{93} \gg$.

Si l'usage de poison est très fréquent (tous les libertins le pratiquent ${ }^{94}$ ), le chocolat n'en représente qu'une infime partie, comme dans cet exemple tiré de l'Histoire de Juliette. Saint-Fond a fait embastiller la marquise de Rose pour lui extorquer cent mille écus et abuser de son jeune fils :

Premièrement, je veux bien prendre les cent mille écus de la comtesse, je veux bien foutre son fils ; mais pour sa sortie de la Bastille, elle ne se fera jamais que dans un coffre. [...] la comtesse ignore que si elle perdait son fils, quoique son parent de fort loin, je serais pourtant son seul héritier; dans un mois la putain n'existera plus, et quand j'aurai bien

\footnotetext{
${ }^{88}$ Sade, Les Infortunes de la vertu, [1787] 1995, p. 34 sqq.; versions modifiées dans Justine ou les Malheurs de la vertu, [1791] 1995, p. 187-192 et La Nouvelle Justine, [1799] 1995, p. 499-510.

89 Sade, Les Infortunes de la vertu [1787] 1995, p. 38 sqq.

90 Voir Béatrice Didier, Sade : une écriture du désir, 1976, p. 85 sq.

${ }^{91}$ Sade, Justine ou les Malheurs de la vertu, [1791] 1995, p. 192 sqq.

${ }^{92}$ Sade, La Nouvelle Justine, [1799] 1995, p. 510.

${ }^{93}$ Ibid., p. 512.

${ }^{94}$ Cf. Mladen Kozul, Le Corps dans le monde. Récits et espaces sadiens, 2005, p. 153.
} 
foutu monsieur son cher fils cette nuit, nous lui ferons prendre une tasse de chocolat demain matin, qui détournera bientôt en ma faveur, l'héritage qu'il aurait pu faire ${ }^{95}$.

Le plan est mis à exécution par Juliette :

Sur les 11 heures le chocolat se servit; j'eus soin [...] de jeter ce qui devait assurer l'héritage à mon amant; et lui par un insigne raffinement de cruauté, voulut pendant que je préparais le poison du fils, donner l'ordre au commandant de la Bastille, d'administrer celui de la mère.

« Allons », me dit Saint-Fond, dès qu'au moyen de nos fourberies, la mort se fut introduite dans les veines de ce malheureux enfant, «allons, voilà ce que j'appelle une bonne matinée $[\ldots]^{96}$.

Dans les exemples sadiens, le récit se limite à l'essentiel, la mise en scène est secondaire. Aussi les toposèmes y apparaissent-ils de façon limitée : les motivations (vénales et ouvertement sadiques) sont développées, le choix du poison $\left(\operatorname{arsenic}^{97}\right)$ ou d'un somnifère (stramonium ${ }^{98}$ ), parfois l'effet sur le corps de la victime. L'originalité de Sade est de se focaliser sur les empoisonneurs et de faire entrer ce topos dans son système de pensée : « Le poison fusionne la vengeance, l'érotisme et la jouissance du pouvoir ${ }^{99} »$. Pensant à ce meurtre qu'il va bientôt réaliser, Saint Fond éprouve une excitation sexuelle peu commune qui décuple la «perfide joie » de la vengeance ${ }^{100}$. Sade va cependant plus loin, comme le constate Mladen Kozul, puisque « la symptomatologie du corps libertin confond les signes de l'empoisonnement et de la jouissance ${ }^{101} »$ : décharge et agonie se résolvent en convulsions. Le chocolat est alors une gourmandise, un « raffinement» supplémentaire dans la scélératesse.

\section{Conclusion}

Que permettent ces exemples d'un point de vue satorien? Peut-on dégager des topö̈ narratifs liés à la consommation de chocolat? Première possibilité, minimaliste et peu satisfaisante «prendre son chocolat ». Deuxième possibilité : « inviter à prendre le chocolat » qui mettrait alors l'accent sur la dimension sociale de ce rituel et présente l'avantage de fonctionner pour un grand nombre d'occurrences. Troisième possibilité : « éprouver plaisir en

\footnotetext{
${ }^{95}$ Sade, La Nouvelle Justine, [1799] 1995, p. 540 sq.

${ }^{96}$ Ibid., p. 541.

${ }^{97}$ Sade, Histoire de Juliette, [1797] 1998, p. 541.

${ }^{98}$ Ibid., p. 726. Le stramonium est administré à Minski dans son chocolat.

${ }^{99}$ Mladen Kozul, op. cit., p. 153.

100 Sade, Histoire de Juliette, [1797] 1998, p. 541.

${ }^{101}$ Mladen Kozul, op. cit., p. 154.
} 
dégustant son chocolat 》 voire «éprouver plaisir lié au chocolat 》 qui associerait alors les différentes perceptions des sens, éventuellement dans un contexte libertin.

Pourrait-on de même proposer un topos lié au chocolat empoisonné ? Plusieurs formulations s'avèrent peu satisfaisantes car elles ne prennent en compte que certains toposèmes, ainsi « boire un chocolat empoisonné » ne dit rien de la préparation et ne recouvre guère les tentatives qui ont échoué ; « préparer un chocolat empoisonné » ne dit rien de l'issue de la situation ; « tenter d'empoisonner avec du chocolat » sous-entend l'échec de l'entreprise. De même, la focalisation sur le poison occulte la possibilité de dissimuler d'autres substances. Reste alors «(tenter d') administrer poison ou narcotique dans chocolat » qui s'appliquerait à plusieurs de nos extraits (Candide, Félicia ou Mes fredaines, Les Infortunes de la vertu ${ }^{102}$, l'Histoire de Juliette). L'on peut dégager également certains toposèmes : vengeance, durée de l'agonie, les convulsions de la victime. D'autres occurrences sont évidemment nécessaires pour élargir et nuancer ces premières ébauches théoriques.

Plutôt que de conclure sur la richesse de l'exemple du chocolat qui lie histoire des choses banales, imaginaire littéraire et pharmacologie, laissons le dernier mot au Mercure galant. L'article consacré à la « science du chocolat ${ }^{103}$ » se termine en effet sur une chanson, intitulée « Le Chocolat» et chantée «sur l'air de Joconde ${ }^{104}$ » qui heureusement ne contient aucun poison :

Le vin est pour le tiers état / il déroge à noblesse, / La loi ne permet qu'au pied plat, / les risques de l'ivresse. / Faisons mousser le chocolat, / Qu'on m'en donne une prise, / C'est friandise de Prélat, / Et la loi l'autorise

[...] L'un de son travail rebuté / Par lui seul y résiste, / À l'autre de l'oisiveté, / Il rend l'ennui moins triste. / Par lui le dîner évité, / Grand souper autorisé, / L'un en prend par sobriété, / L'autre par gourmandise.

Par le chocolat répété, / L'appétit se déroute, / Son éloge aussi trop chanté, / Dégouterait sans doute. Chers friands encore un couplet, / Passez-le moi de grâce / Moy d'un chocolat à souhait, / Je vous passe une tasse.

Il donne ou guérit les vapeurs, / Selon qu'on le compose, / Vous Médecins ou Directeurs, / Sachez-en bien la dose. / Vous y ferez pour le repos, / Des femmes ou des filles, / Dominer les froids cacaos, / Ou les chaudes vanilles ${ }^{105}$.

\footnotetext{
${ }^{102}$ Le même topos dans les réécritures romanesques doit-il être comptabilisé une ou plusieurs fois ?

${ }^{103}$ Voir supra note 2.

${ }^{104}$ Mercure galant, novembre 1711, p. 23.

${ }^{105}$ Ibid., p. 23-27.
} 


\section{Illustrations}

Fig. 1 : François Boucher, Le Déjeuner, 1739, $81 \mathrm{~cm} \mathrm{x} 65 \mathrm{~cm}$, huile sur toile, Paris, Musée du Louvre.

Fig. 2 : Jean-Étienne Liotard, La Belle Chocolatière, 1743, 82,5 cm x 52,5 cm, pastel sur parchemin, Dresde, Gemäldegalerie Alte Meister.

Fig. 3 : Jean-Baptiste Charpentier, Le Vieux, La Famille du duc de Penthièvre en 1768 dit La Tasse de Chocolat, 1768, $176 \mathrm{~cm}$ x $256 \mathrm{~cm}$, huile sur toile, Versailles, châteaux de Versailles et de Trianon.

Fig. 4 : Jean-Baptiste Le Prince, La Crainte, 1769, $50 \mathrm{~cm}$ x $64 \mathrm{~cm}$, huile sur toile, Toledo, Museum of Art.

\section{Bibliographie}

\section{Euvres et sources}

Mercure galant, novembre 1711.

ANONYME, Le Petit-fils d'Hercule dans Romanciers libertins du XVIIIe siècle, t. II, Paris, Gallimard (Bibliothèque de la Pléiade), 2005, [P. Wald-Lasowski (éd.)], p. 1073-1137.

ANONYME, Les Mémoires de Suzon dans Romanciers libertins du XVIIIe siècle, t. II, Paris, Gallimard (Bibliothèque de la Pléiade), 2005, [P. Wald-Lasowski (éd.)] p. 873-971.

AULNOY (d'), Relation du voyage d'Espagne, Paris, chez Claude Barbin, 1691, 3 vol.

BEAUMARCHAIS, Mémoires contre M. Goëzmann, Euvres complètes, t. 3, Burne, [1774] 1828.

BERNARDIN DE SAINT-PIERRE, Voyage à l'île de France, Euvres posthumes, t. 1, Paris, Ledentu, 1840.

DIDEROT, Denis, La Religieuse dans Contes et romans, Paris, Gallimard (Bibliothèque de la Pléiade), 2004, [M. Delon (éd.)], p. 239-415.

DIDEROT, Denis, Lettre sur les sourds et muets à l'usage de ceux qui entendent et qui parlent dans Euvres complètes, t. IV, Le nouveau Socrate, Idées II, Paris, Hermann, 1978.

DIDEROT, Denis et LE ROND D’ALEMBERT, Jean (éds.), Encyclopédie, ou dictionnaire raisonné des sciences, des arts et des métiers, University of Chicago, ARTFL Encyclopédie Project [R. Morrissey et G. Roe (éds.)], automne 2017.

URL : http://encyclopedie.uchicago.edu/.

DULAURENS, Le Compère Mathieu ou les Bigarrures de l'esprit humain, Paris, Honoré Champion, 2012 [D. Gambert (éd.)]. 
FOUGERET DE MONBRON, Margot la ravaudeuse [1750] dans Romanciers libertins du XVIIIe siècle, t. I, Paris, Gallimard (Bibliothèque de la Pléiade), 2000, [P. Wald-Lasowski (éd.)], p. 801-866.

GODARD D'AUCOUR, Thémidore dans Romanciers libertins du XVIIIe siècle, t. I, Paris, Gallimard (Bibliothèque de la Pléiade), 2000, [P. Wald-Lasowski (éd.)], p. 501-586.

GOULIN, Jean et LABEYRIE, (prénom non indiqué), Dictionnaire raisonné universel de matière médicale, Paris, chez François Didot, 1773, 4 vol.

LÉMERY, Nicolas, Traité universel des drogues simples, Paris, chez Laurent d'Houry, 3e édition, 1723.

LÉMERY, Louis, Traité des aliments où l'on trouve par ordre et séparément la différence et le choix qu'on doit faire de chacun d'eux en particulier, les bons et les mauvais effets qu'ils peuvent produire, les principes en quoi ils abondent..., Paris, J.-B. Cusson \& P. Witte, 1702, in-8 ; 2e éd., Paris, P. Witte, 1705.

LESAGE, Le Diable boiteux dans Romanciers du XVIIIe siècle, t. I, Paris, Gallimard (Bibliothèque de la Pléiade), [1726] 1987, [Étiemble (éd.)], p. 269-494.

LESAGE, Histoire de Gil Blas de Santillane dans Romanciers du XVIIIe siècle, t. I, Paris, Gallimard (Bibliothèque de la Pléiade), 1987, [Étiemble (éd.)], p. 495-1201.

LESAGE, Histoire de Guzman d'Alfarache, Berquet, 1825, 4 vol.

LOUVET DE COUVRAY, Six semaines de la vie de Faublas dans Romanciers du XVIIIe siècle, t. II, Paris, Gallimard (Bibliothèque de la Pléiade), 1965, [Étiemble (éd.)], p. 721-856.

MIRABEAU, Le Libertin de qualité, ou Ma conversion, Paris, Bibliothèque des Curieux, [1783] 1911.

NERCIAT, Félicia ou Mes fredaines dans Romanciers libertins du XVIIIe siècle, t. II, Paris, Gallimard (Bibliothèque de la Pléiade), [1775] 2005, [P. Wald-Lasowski (éd.)], p. 591-872. POMET, Pierre, Histoire générale des drogues traitant des plantes, des animaux \& des Mineraux, ouvrage enrichy de plus de quatre cent Figures en Taille-douce tirées d'après Nature; avec un discours qui explique leurs differens Noms, les Pays d'où elles viennent, la maniere de connoître les Veritables d'avec les Falsifiées, \& leurs proprietez, où l'on découvre l'erreur des Anciens \& des Modernes ; Le tout très utile au Public, Paris, chez Estienne Ducastin, 1694.

RESTIF DE LA BRETONNE, L'Anti-Justine, Paris, Le Cercle Poche, [1798] 2014.

SADE, Les Infortunes de la vertu, Justine ou les Malheurs de la vertu, La Nouvelle Justine dans Euvres, t. II, Paris, Gallimard (Bibliothèque de la Pléiade) 1995, [M. Delon (éd.)] 
SADE, Juliette dans CEuvres, t. III, Paris, Gallimard (Bibliothèque de la Pléiade) 1998, [M. Delon (éd.)], p. 179-1262.

SÉVIGNÉ, Mme de, Correspondance, Paris, Gallimard (Bibliothèque de la Pléiade), 19721978, 3 vol. [R. Duchêne et J. Duchêne (éds.)].

VARENNE, Jacques de, Mémoires du chevalier de Ravanne, Aux dépends de la Compagnie, 1782, 4 vol.

VOLTAIRE, Candide, Paris, Librairie Générale Française (Le Livre de poche), 1995, [Sylviane Léoni (éd.)].

VOLTAIRE, La Princesse de Babylone dans Romans et Contes, Paris, Gallimard (Bibliothèque de la Pléiade), [1768] 1954 [R. Groos (éd.)], p. 366-432.

Études

BERNARD, Michel, « Madame Bovary ou le danger des sucreries », Romantisme, n¹03, 1999, p. $41-51$.

BERNIER, Marc André, Libertinage et figures du savoir. Rhétorique et roman libertin dans la France des Lumières (1734-1751), Québec/Paris, Presses de 1'Université Laval / L'Harmattan, (Les Collections de la République des Lettres), 2001.

BOKOBZA KAHAN, Michèle, Libertinage et Folie dans le roman du 18 e siècle, Louvain, Éditions Peeters (La République des Lettres), 2000.

BONNET, Jean-Claude, « Le réseau culinaire dans 1'Encyclopédie », Annales. Économies, sociétés, civilisations, vol. 31, n5, 1976, p. 891-914.

BRAUDEL, Fernand, Civilisation matérielle, économie et capitalisme, XVe-XVIIIe siècle, t. 1, Les structures du quotidien : le possible et l'impossible, Paris, Armand Colin, 1979.

COE, Sophie D. et COE, Michael D., Généalogie du chocolat, Paris, Éditions Abbeville, 1998. CORBIN, Alain, Le miasme et la jonquille, Paris, Champs Flammarion, [1982] 2016.

DELON, Michel, « Le détail et l'histoire », dans Claire JAQUIER, Florence LOTTERIE et Catriona SETH (éds.), Destins romanesques de l'émigration, Paris, Desjonquères, 2007, p. 158-168.

DELON, Michel (éd.), Dictionnaire européen des Lumières, Paris, Presses Universitaires de France, 1997.

DIDIER, Béatrice, Sade : une écriture du désir, Paris, Denoël / Gonthier, 1976. 
DUECK Evelyn et VUILLEMIN Nathalie (éds.), " Der Augen Blödigkeit », Sinnestäuschungen, Trugwahrnehmungen und visuelle Epistemologie im 18. Jahrhundert, Heidelberg, Winter, 2016.

DUECK Evelyn et VUILLEMIN Nathalie (éds.), Entre l'œil et le monde : dispositifs d'une nouvelle épistémologie visuelle dans les sciences de la nature (1740-1840), éd. Épistémocritique, 2017, en ligne. URL : https://epistemocritique.org/entre-loeil-mondedispositifs-dune-nouvelle-epistemologie-visuelle-sciences-de-nature-1740-1840/

EVANS, Michael R., Inventing Eleanor. The Medieval and Post-Medieval Image of Eleanor of Aquitaine, Londres / Oxford, Bloomsbury, 2014.

HERRERO Isabel et VAZQUEZ Lydia, « Types nationaux européens dans des œuvres de fiction françaises (1750-1789) », Dix-huitième Siècle, n²5, 1993, p. 115-127.

HOFFMANN, Kathryn A., « Palimpsests of Knowledge, Feast of Words : Antoine Furetière's Dictionnaire universel », Cahiers du dix-septième - An Interdisciplinary Journal, Vol. VII, $\mathrm{n}^{\circ} 1,1997$, p. 47-59.

KOZUL, Mladen, Le Corps dans le monde. Récits et espaces sadiens, Louvain, Peeters, 2005.

LAFON, Henri, Les Décors et les choses dans le roman français du dix-huitième siècle de Prévost à Sade, Oxford, Voltaire Foundation, 1992.

LEVIN, D. M., Modernity and the Hegemony of Vision, Berkeley, University of California Press, 1993.

PATERNOTTE, Stéphanie et LABRUDE, Pierre, " Le chocolat dans quelques ouvrages français de pharmacie et de médecine des XVIIe, XVIIIe et XIXe siècles. Ses effets fastes et néfastes avérés ou supposés ", Revue d'histoire de la pharmacie, vol. 91, n³38, 2003, p. 197-210.

PEETERS, Alice, « Boire le chocolat », Terrain, $\mathrm{n}^{\circ} 13$, octobre 1989, URL : http://journals.openedition.org/terrain/2959.

PEETERS, Alice, « Controverses sur les vertus du cacao et les manières de préparer le chocolat (XVIe-XVIIIe siècle) », Journal d'agriculture traditionnelle et de botanique appliquée, vol. 26, n³-4, Juillet-décembre 1979, p. 201-216.

PUCCINI-DELBEY, Géraldine, Le Débat des cinq sens de l'Antiquité à nos jours, Pessac, PUB, 2014.

RAMBOURG, Patrick, « De la consommation des boissons exotiques aux XVIIe et XVIIIe siècles ", Thé, café ou chocolat? Les boissons exotiques à Paris au XVIIIe siècle, catalogue de l'exposition au Musée Cognacq-Jay, Paris musées, 2015, p. 47-61. 
RIPOLL, Élodie, Penser la couleur en littérature. Explorations romanesques des Lumières au réalisme, Paris, Classiques Garnier (Confluences), 2018.

RUSTIN, Jacques, « Définition et explicitation du roman libertin des Lumières », Travaux de linguistique et de littérature, XVI, 2, 1978, p. 26-34.

SCHÖCH, Christof, La Description double dans le roman des Lumières, Paris, Classiques Garnier (L'Europe des Lumières), 2011.

SÉRET, Guillaume, « Des porcelaines pour sublimer le goût », Thé, café ou chocolat ? Les boissons exotiques à Paris au XVIIIe siècle, catalogue de l'exposition au Musée CognacqJay, Paris musées, 2015, p. 85-95.

SMITH, Mark M., Sensory History, Oxford \& New York, Berg, 2007.

STALNAKER, Joanna, The Unfinished Enlightenment. Description in the Age of the Encyclopedia, Ithaca \& Londres, Cornell UP, 2010. 